\title{
The 'fibrinolytic potential' as a simple measure of spontaneous fibrinolysis
}

\author{
R. CHAKRABARTI AND G. R. FEARNLEY
}

From the Gloucestershire Royal Hospital, Gloucester

SYNOPSIS Natural or spontaneous fibrinolytic activity of the blood is due to a labile activator which is stabilized by fibrin formation. Current methods of measuring spontaneous fibrinolysis require either low temperature centrifugation when plasma is used or photography when diluted whole blood is used and neither is available in the average laboratory. A method of measuring fibrinolytic activity in blood, the 'fibrinolytic potential', which requires only simple apparatus, is described. It is found to correlate well with the dilute blood clot lysis time, and should be of value for investigating the hitherto neglected problem of spontaneous fibrinolytic activity in occlusive vascular disease.

Measurement of the natural or spontaneous fibrinolytic activity of blood is hampered by the lability of the factor responsible (Fearnley, Revill, and Tweed, 1952), and centrifugation at a low temperature is essential for methods which employ plasma, including the euglobulin clot lysis time. A simpler method is the lysis time of a $10 \%$ dilution of blood in phosphate buffer clotted with thrombin (Fearnley, Balmforth, and Fearnley, 1957) but this has the disadvantage of requiring photographic recording of the end-point (Lackner and Goosen, 1959).

For multiple determinations a simple method employing blood rather than plasma and giving a clear-cut end-point without photography is needed. The method described here correlates well with the dilute blood clot lysis time, and requires neither centrifugation at low temperatures nor photography. It depends on the fact that dilution favours fibrinolysis, and per contra that increasing the amount of blood in the test prolongs the lysis time. When a series of increasing blood concentrations is set up and clotted with thrombin, the curve relating lysis time and blood concentration is assumed to reflect the balance between the activator level and the inhibitory capacity of the blood sample. This balance, termed by us the 'fibrinolytic potential', is measured by the highest concentration of blood showing lysis after a set period of incubation. The fibrinolytic potential is described and illustrated.

\section{METHOD}

Blood obtained by venepuncture is immediately cooled in melting ice. Five $\mathrm{ml}$. blood is diluted with an equal Received for publication 17 April 1961. volume of ice-cold phosphate buffer at $p \mathbf{H ~ 7 \cdot 4 , ~ M . ~} 0 \cdot 12$ (for preparation of buffer, see Fearnley et al., 1957). Ten $1.90 \mathrm{ml}$. volume dilutions of blood in phosphate buffer are made in test-tubes standing in melting ice, the dilutions being such that the final concentration of blood in the test (volume $2.0 \mathrm{ml}$.) ranges from 5 to $27.5 \%$, the blood concentration interval between the tubes being $2.5 \%$. To each tube is added $0.1 \mathrm{ml}$. physiological saline containing 5 units of Parke Davis topical thrombin, each tube being shaken after adding the thrombin. After 15 minutes the tubes are placed in a water-bath at $37^{\circ} \mathrm{C}$. Five to 10 minutes later each tube is briskly rotated between the palms of the hands to ensure freedom of retraction. The test is read after 12 hours' incubation, the highest concentration of blood to show complete lysis being the endpoint. For the purposes of this investigation the progress of lysis was recorded photographically as described by Fearnley, Ferguson, Chakrabarti, and Vincent (1960). After the test has been set up, in order to choose a convenient time for incubation, the tubes may be stored in the refrigerator for up to six hours before being placed in the water-bath.

\section{RESULTS}

In Fig. 1 is shown the relationship between percentage blood concentration and time of lysis in six $\omega$ subjects. Subjects A, B, and $\mathrm{C}$ were healthy males, and $D, E$, and $F$ were patients with atherosclerosis. It can be seen that the shorter the lysis time of the $10 \%$ blood clot, and hence the greater the fibrinolytic activity of the blood sample, the higher is the percentage lysis after 12 hours' incubation.

In Fig. 2 is shown the correlation between the $10 \%$ blood clot lysis times and the percentage lysis at 12 hours in 35 normal and atherosclerotic subjects. Some degree of scatter results from the arbitrary 


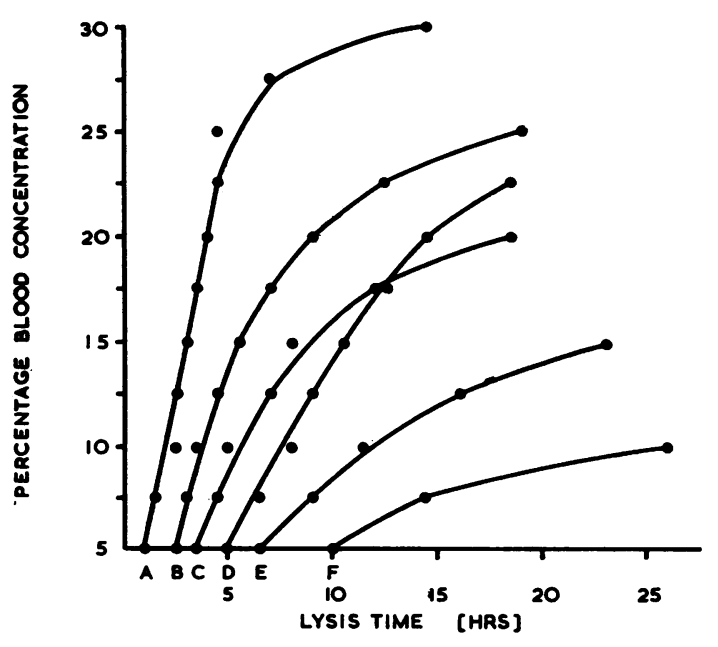

FIG. 1. The relationship between percentage blood concentration and time of lysis in six subjects. Subjects $A, B$, and $C$ were healthy males and $D, E$, and $F$ male patients with atherosclerosis.

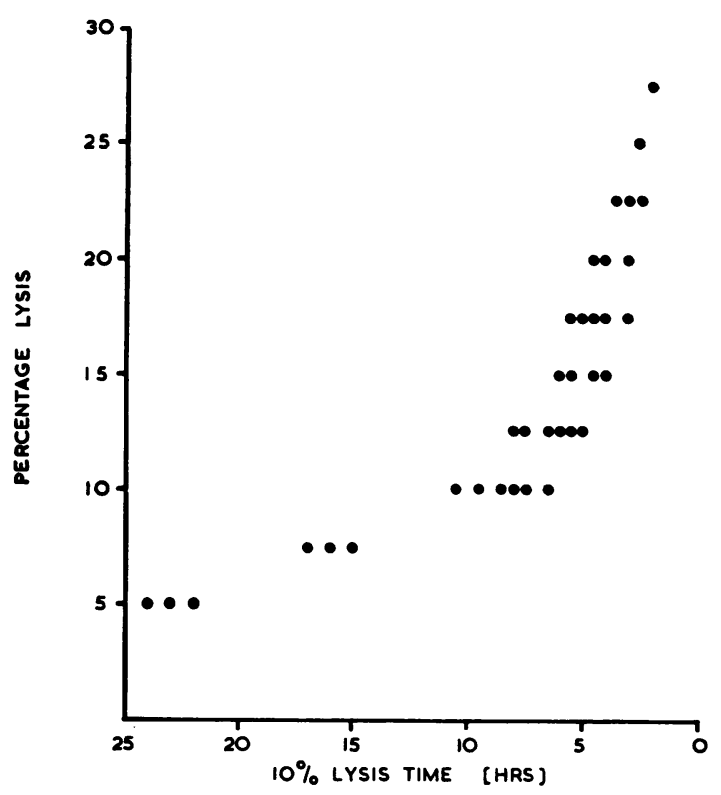

FIG. 2. The correlation between the $10 \%$ blood clot lysis times and the percentage lysis at 12 hours ("fibrinolytic potential') in 35 subjects.

interval between the percentage concentrations but it is evident that both tests measure the same phenomenon. Their relation is exponential.

To determine the utility of the fibrinolytic potential as a measure of spontaneous fibrinolysis, diurnal variability (Fearnley et al., 1957; Buckell and Elliott, 1959) was compared using this method and the $10 \%$ blood clot lysis time. Ten female in-patients with various diseases were studied, blood being obtained in the fasting state between 8 and $8.30 \mathrm{a} . \mathrm{m}$. and again between 5 and 5.30 p.m. on the same day. The results (Table I) indicate that the fibrinolytic potential reflects the diurnal changes of blood fibrinolytic activity shown by the $10 \%$ blood clot lysis time.

\section{TABLE I}

DIURNAL VARIATION OF FIBRINOLYSIS SHOWN BY THE FIBRINOLYTIC POTENTIAL AND $10 \%$ BLOOD CLOT LYSIS TIME

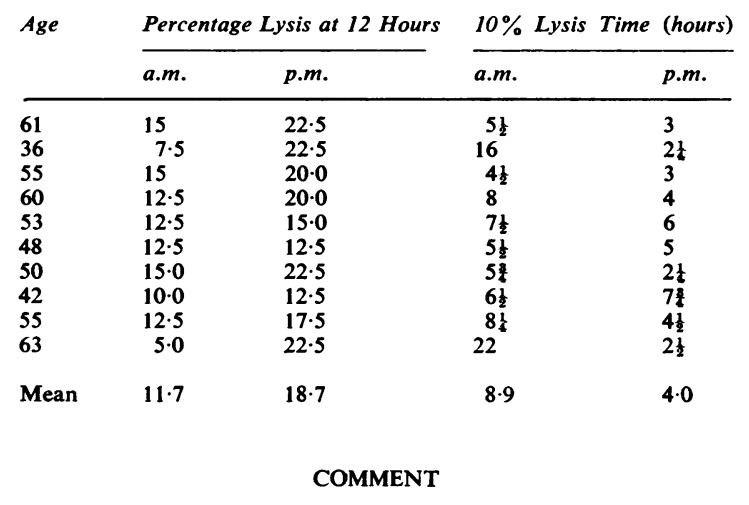

Spontaneous fibrinolytic activity has been shown to be due to the presence in freshly obtained blood of a plasminogen activator, whose lability is caused by the production of an anti-activator in shed blood (Flute, 1959). The formation of fibrin protects the activator from inactivation, presumably because it is adsorbed to fibrin (Fearnley, 1953; Fearnley and Ferguson, 1958). A concept of how the spontaneous fibrinolytic activity of blood may function as a remover of deposited fibrin and hence as an antithrombotic mechanism has been outlined (Fearnley, 1961). Little information about spontaneous fibrinolysis in healthy and atherosclerotic people is available, and the number of patients studied for this paper is insufficient for any conclusion. It is hoped, however, that a more extensive application of simple methods such as the one described may permit the evaluation of spontaneous fibrinolytic activity in the multifactorial problem of thrombo-embolic disease.

Current methods of measuring spontaneous fibrinolysis are crude and do not differentiate between inhibition on the one hand and activator level on the other, although it is possible that the euglobulin clot lysis time measures uninhibited activity. Until more 
is known of spontaneous fibrinolysis, and it seems likely that the system may prove to be as complex as blood coagulation, there is much information to be obtained with the relatively crude tests at present available which give an overall measure of fibrinolysis comparable to the clotting time of blood Without special equipment, however, current methods cannot be performed in the average laboratory. Measuring the fibrinolytic potential of blood requires no special apparatus, yields a sharp end-point, and appears to correlate reasonably well with the $10 \%$ blood clot lysis time.

This study was supported by the South West Regional Hospital Board and Messrs. Pfizer Ltd. (Harvey Pharma- ceuticals), Dr. Chakrabarti's salary as research registrar being paid by Messrs. Pfizer.

We wish to thank Dr. E. N. Davey for generously giving us laboratory space and Miss Esther Gronow and Mr. John Evans for efficient technical assistance.

\section{REFERENCES}

Buckell, M., and Elliott, F. A. (1959). Lancet, 1, 660.

Fearnley, G. R. (1953). Nature (Lond.), 172, 544.

(1961). Lancet, 1, 992.

Balmforth, G., and Fearnley, E. (1957). Clin. Sci., 16, 645.

and Ferguson, J. (1958). Ibid., 17, 555.

- Chakrabarti, R., and Vincent, C. T. (1960). Lancet, 1, 184. Revill, R., and Tweed, J. M. (1952). Clin. Sci., 11, 309.

Flute, P. Proceedings of the 7th European Congress of Haematology. London, 1959. Karger, Basle. In press.

Lackner, H., and Goosen, C. C. (1959). Acta haemat. (Basel), 22, 58. 